\title{
New insights into the impact of neuro-inflammation in rheumatoid arthritis
}

\author{
Nicholas R. Fuggle ${ }^{1}$, Franklyn A. Howe ${ }^{2}$, Rachel L. Allen ${ }^{1}$ and Nidhi Sofat ${ }^{1 *}$ \\ 1 Institute of Infection and Immunity, St. George's University, London, UK \\ ${ }^{2}$ Neuroscience Research Centre, Institute of Cardiovascular and Cell Sciences, St. George's University, London, UK
}

\section{Edited by:}

Luba Sominsky, RMIT University,

Australia

\section{Reviewed by:}

Masaaki Murakami, Hokkaido

University, Japan

Paola Patrignani, "G. d'Annunzio"

University, Italy

*Correspondence:

Nidhi Sofat, Institute of Infection and Immunity, St. George's University,

Blackshaw Road, London

SW17 0QT, UK

e-mail:nsofat@sgul.ac.uk
Rheumatoid arthritis (RA) is considered to be, in many respects, an archetypal autoimmune disease that causes activation of pro-inflammatory pathways resulting in joint and systemic inflammation. RA remains a major clinical problem with the development of several new therapies targeted at cytokine inhibition in recent years. In RA, biologic therapies targeted at inhibition of tumor necrosis factor alpha (TNF $\alpha$ ) have been shown to reduce joint inflammation, limit erosive change, reduce disability and improve quality of life. The cytokine TNF $\alpha$ has a central role in systemic RA inflammation and has also been shown to have pro-inflammatory effects in the brain. Emerging data suggests there is an important bidirectional communication between the brain and immune system in inflammatory conditions like RA. Recent work has shown how TNF inhibitor therapy in people with RA is protective for Alzheimer's disease. Functional MRI studies to measure brain activation in people with RA to stimulus by finger joint compression, have also shown that those who responded to TNF inhibition showed a significantly greater activation volume in thalamic, limbic, and associative areas of the brain than non-responders. Infections are the main risk of therapies with biologic drugs and infections have been shown to be related to disease flares in RA. Recent basic science data has also emerged suggesting that bacterial components including lipopolysaccharide induce pain by directly activating sensory neurons that modulate inflammation, a previously unsuspected role for the nervous system in host-pathogen interactions. In this review, we discuss the current evidence for neuro-inflammation as an important factor that impacts on disease persistence and pain in RA.

Keywords: neuroinflammation, rheumatoid arthritis, tumor necrosis factor alpha, neuroimaging, infection, lipopolysaccharide

\section{INTRODUCTION}

Rheumatic diseases include some of the most common chronic disorders worldwide. Of these, rheumatoid arthritis (RA) is considered to be, in many respects, an archetypal autoimmune disease (Feldmann and Maini, 2010). RA causes activation of proinflammatory pathways resulting in joint and systemic inflammation and remains a major clinical problem (McInnes and Schett, 2011). Treatments with immune-modulatory drugs, including biologic therapies, have revolutionized its management. New

Abbreviations: ACPA, Anti-Citrullinated Protein Antibody; AD, Alzheimer's Disease; BBB, blood-brain barrier; Cho, Choline; Cr, Creatine; COX, CycloOxygenase; CS, Corticosteroids; CSSERP, Chemo-Somatosensory Event-Related Potentials; DAMPs, damage associated molecular patterns; DMARD, DiseaseModifying Anti-Rheumatic Drug; DRG, Dorsal Root Ganglia; DTI, Diffusion Tensor Imaging; ESR, Erythrocyte Sedimentation Rate; fMRI, Functional Magnetic Resonance Imaging; ICAM, intercellular adhesion molecule; IFN- $\beta$, Interferon- $\beta$; IL, Interleukin; ISG, Interferon-Stimulating Genes; LPS, lipopolysaccharide; MRI, Magnetic Resonance Imaging; MRS, Magnetic Resonance Spectroscopy; NAA, NAcetyl Aspartate; PADIV, Peptidyl Arginine Deaminase type IV; RA, Rheumatoid Arthritis; RNA, ribonucleic acid; SE, Shared Epitope; SNP, Single Nucleotide Polymorphism; TACE, Tumor necrosis factor alpha converting enzyme; Th, T helper cell; TLR, Toll-like receptor; TNF $\alpha$, tumor necrosis factor alpha; WM, White Matter. therapies target relevant cytokines such as tumor necrosis factor alpha $(\mathrm{TNF} \alpha)$ and immune cells such as B cells. In RA, biologics have been shown to reduce joint inflammation, limit erosive change, reduce disability and improve quality of life (Scott et al., 2010). Biologics are mainly co-administered with disease-modifying drugs such as methotrexate when the latter are found to achieve insufficient disease control on their own (Firestein, 2003). Recent work using functional neuroimaging has suggested that TNF inhibitors may also reduce central nervous system activity related to inflammation-induced pain in people with RA using brain neuroimaging (Rech et al., 2013). Classically, the term "neuro-inflammation" has implicated the activation of microglia and astroglia which in turn activate the expression of pro-inflammatory cytokines and chemokines in a variety of conditions including Alzheimer's disease (AD) (O'Callaghan et al., 2008). However, the trigger(s) for activation of a potential neuro-inflammatory process and furthermore its measurement in clinical disease is not always straightforward to decipher. Several groups have utilized animal models to discover that although certain features of inflammation are typical for the pro-inflammatory response systemically e.g., 
interleukin-6 (IL-6), interferon- $\beta$ (IFN $\beta$ ), these are not observed in the brain, but activation of $\mathrm{TNF} \alpha$ does appear to maintain pro-inflammatory activity in the brain as it does systemically (Skelly et al., 2013; Thomson et al., 2014). Some of the basic science observations can be extended to RA, where Chou et al. (2010) observed that people with RA receiving TNF inhibitor treatment (infliximab, etanercept, adalimumab) showed a reduction in the risk of developing $\mathrm{AD}$ compared to controls (Chou et al., 2010). The risk of AD was not affected by exposure to other Disease-Modifying Anti-Rheumatic Drugs (DMARDs) used in RA. Recent work by Detrait et al. (2014) has also shown that peripheral administration of TNF inhibitors in mice counteracts the amyloid-induced increase of hippocampal TNF $\alpha$ levels and memory deficits in mice. In this review, we explore the theme of neuro-inflammation in RA. Although as yet, the cause-and-effect relationship between infection, autoimmunity, neural correlates and RA disease activity are not understood mechanistically, we review the current status of the field.

\section{CURRENT CONCEPTS IN THE PATHOGENESIS OF RHEUMATOID ARTHRITIS}

RA is an immune-mediated pro-inflammatory disease, often resulting in chronic disability, early mortality, systemic complications and high socioeconomic burden on society as a whole (McInnes and Schett, 2011). There has been a vast improvement in our understanding of this condition over the last few decades, resulting in improved treatments which are now initiated early in order to maximize and maintain disease remission (van Vollenhoven, 2010).

Risks for developing RA involve a complex interplay between genotype, environment and lifestyle factors such as smoking (Mahdi et al., 2009). Twin studies have shown a concordance rate of 15-30\% among monozygotic twins and 5\% among dizygotic twins (MacGregor et al., 2000). Genome-wide association analyses have uncovered immune regulatory factors that may underlie the disease; including PTPN22 among the Single Nucleotide Polymorphisms (SNPs) identified (Wellcome Trust Case Control Consortium, 2007). An association with HLADRB1 has been established for RA patients who are positive for rheumatoid factor or anti-citrullinated peptide antibodies (ACPA) (Gregersen et al., 1987). In keeping with the role of HLA-DRB1 in antigen presentation, a number of studies over the last 2 decades have shown that auto-reactive immune responses are mediated by $\mathrm{T}$-cell repertoire selection, antigen presentation or changes in peptide affinity (Panayi, 2006). The shared epitope (SE), carried by the vast majority of RA patients, is a 5-aa sequence motif in the third allelic hypervariable region of the HLA-DR $\beta$ chain. Proposed explanations for the link between RA and the SE include molecular mimicry of the SE by microbial proteins, increased $\mathrm{T}$ cell senescence induced by SE-containing HLA molecules and a potential pro-inflammatory signaling function that is unrelated to the role of the SE in antigen recognition (Weyand and Goronzy, 1990; De Almeida et al., 2010).

Gene-environment interactions are also important in RA development. Smoking and other environmental risks to the lung such as silica exposure, increase the risk of RA in people with susceptibility HLA-DR4 alleles (Symmons et al., 1997; Klareskog et al., 2008). Smoking and HLA-DRB1 alleles synergistically increase one's risk of developing the anti-citrullinated protein antibodies (ACPA) that are present in the majority of patients with RA (Klareskog et al., 2008). It has therefore been proposed that environmental stress in the lung or other mucosal surfaces may promote post-translational modifications through activation of peptidyl arginine deiminase, type IV (PADIV), resulting in citrullination of mucosal proteins. Loss of tolerance to the neoepitopes generated by citrullination can be detected clinically in people with RA by the ACPA response (Vincent et al., 1999).

It has long been recognized that infectious agents such as cytomegalovirus, E. coli, Epstein Barr virus, parvovirus, and proteus species may play a role in RA. Recently, the oral pathogen Porphyromonas gingivalis has been implicated in the pathogenesis of RA (Mikuls et al., 2014). Products of infectious agents e.g., heat shock proteins and enzymes responsible for citrullination have been shown in several models to induce immune reactivity. For example, several citrullinated self-proteins can be identified in ACPA assays, including alpha enolase, keratin, fibrinogen, fibronectin, collagen, and vimentin (van der Woude et al., 2010). Although unifying mechanisms for the link between infection and RA autoimmunity are not entirely established, the theory of molecular mimicry has been proposed (van Heemst et al., 2014). The formation of immune complexes during infection may trigger the induction of rheumatoid factor, which is a high affinity autoantibody against the Fc portion of immunoglobulin, often used in the diagnosis of RA (De Rycke et al., 2004). A link has been described between RA and periodontal disease: Porphyromonas gingivalis produces PAD1V which can promote citrullination of mammalian proteins (Wegner et al., 2010). Recently, the gastrointestinal microbiome has also been implicated in the development of autoimmunity (Scher et al., 2012).

Emerging data suggests there is an important impact of bidirectional communication between the brain and immune system that has a significant impact on RA symptoms. The onset of RA is associated with adverse life events and infectious triggers, where links between the hypothalamic-pituitary-adrenal axis and autoimmunity have been shown (Sokka et al., 2009; Capellino et al., 2010). The impact of external factors such as infections and their impact on disease activity in RA including joint pain/swelling will be discussed further in the following sections. One feature of established RA includes the presence of rheumatoid factor, which is an IgM complex of antibodies to IgG found circulating in RA (Mellor, 1959). Cerebrospinal fluid rheumatoid factor has been demonstrated in case reports of central nervous system manifestations of RA. (Markenson et al., 1979; Inan et al., 2011) Recent studies suggest that circulating immune complexes can elicit a neuro-inflammatory response in the brain. Teeling et al. (2012) showed that in the presence of antigen, antibodies can lead to local immune complexmediated inflammatory reaction in the brain parenchyma and directly induce local tissue damage through the recruitment and activation of microglia through $\mathrm{Fc} \gamma$ receptors (Teeling et al., 2012). 


\section{THE ROLE OF CYCLO-OXYGENASE AND PROSTANOIDS IN NEURO-INFLAMMATION}

Several of the brain effects induced by cytokines such as TNF $\alpha$ are thought to be mediated by regulating the expression of cyclo-oxygenase (COX) enzymes (mainly COX-2) and generation of prostanoids. Under usual physiological conditions, these inflammatory mediators are absent in the brain or found in low levels. After brain injury or insult, there can be induction of pro-inflammatory mediators in astrocytes, microglia, neurons and endothelial cells which results in the development of neuro-inflammatory conditions. Recently, the COX-2 inhibitor celecoxib has been shown to reduce functional connectivity measured by functional MRI in an animal model of osteoarthritis (Upadhyay et al., 2013). In their study, Upadhyay et al. (2013) showed a reduction in blood oxygenation in recognized brain pain centers including the thalamus, hippocampus, periaqueductal gray matter and nucleus accumbens in arthritic rodents treated with celecoxib. Human studies in patients with chronic knee osteoarthritis have also shown that COX inhibitors e.g., valdecoxib, reduce spontaneous brain pain activation signals measured by functional brain MRI (Parks et al., 2011).

One of the earliest studies in human subjects by Ho et al. (2001) demonstrated that neuronal COX-2 expression was increased in post-mortem samples of people with Alzheimer's disease and correlated with clinical progression of dementia (Ho et al., 2001). In Alzheimer's disease, the classical neuropathological changes include deposition of neurofibrillary tangles and $\beta$ amyloid $(A \beta)$. Alzheimer's dementia is typified by activation of microglia and astrocytes in response to $A \beta$ deposition, leading to a release of a variety of factors including the cytokines $\mathrm{TNF} \alpha$ and IL-1 $\beta$, free radicals such as nitric oxide, superoxide and cyclo-oxygenase pathway derived prostanoids (Combs et al., 2001; Hull et al., 2006; Medeiros et al., 2007). Evidence for the activation of the COX pathway has come from neuro-inflammatory diseases that include Alzheimer's disease, Parkinson's disease and multiple sclerosis. For example, in a mouse model of Parkinson's disease, Hunter et al. (2007) showed that celecoxib, a COX-2 inhibitor, inhibited neuronal effects in rats injected with LPS. They found that a number of effects were reduced in rats treated with celecoxib, including inflammation and nigral dopaminergic neuronal loss. It is therefore possible that non-steroidal anti-inflammatory drugs (NSAIDs) such as celecoxib could have an inhibitory effect on neuro-inflammation and could have an impact on delaying neurodegenerative effects.

\section{CAN INFECTION INFLUENCE RHEUMATOID ARTHRITIS PAIN?}

The central nervous system is involved in immune regulation and homeostasis, with neuro-immunological interactions regulating disease development in animal models of arthritis (Chiu et al., 2013). Peripheral inflammation triggered by synovial inflammation in RA stimulates the increased release of cytokines (Taylor, 2014). Cytokine inhibitors administered to patients with a high disease load have also been shown to suppress RA arthritic disease activity (Feldmann and Maini, 2010). More recently, microarray analysis of brain extracts from mice injected peripherally with the gram-negative bacterial stimulus of lipopolysaccharide
(LPS) has indicated an increase in cytokine expression (Thomson et al., 2014). The cytokine up regulation observed was found to be elicited by a Toll-Like Receptor (TLR)-mediated interferon response in mice injected peripherally with LPS (Thomson et al., 2014).

Data has emerged from animal models to suggest that microbial components including LPS can activate sensory neurons directly (Chiu et al., 2013). In their elegant studies, Chiu et al. (2013) applied heat-killed S. aureus to dorsal root ganglia (DRG) sensory neurons and demonstrated an induction of calcium flux and induced action potential firing. S. aureus was found to directly activate nociceptors in the Nav1.8-lineage (Chiu et al., 2013). Other experiments showed that neuronal responses by the Nav 1.8 nociceptor could be activated through heat-killed streptococcus, Listeria monocytogenes, Mycoplasma fermentans, Helicobacter pylori, Pseudomonas aeruginosa, and Escherichia coli. Variations in the pattern of nociceptor responsiveness between bacteria could indicate that strain-specific ligands act through different mechanisms.

\section{THE IMPORTANCE OF TNF AS A PRO-INFLAMMATORY CYTOKINE IN RHEUMATOID ARTHRITIS}

Biologic therapies have been used to treat severe RA since 1997 and have been manufactured to target specific elements of inflammation pathways. They include anakinra, an IL-1 antagonist; abatacept, which down-regulates $\mathrm{T}$ cell activation; rituximab, a chimeric anti-CD20 antibody which reduces B cell activation and infliximab, adalimumab and etanercept which inhibit TNF activity (Malaviya and Ostor, 2012). The European League Against Rheumatism (EULAR) and American College of Rheumatology (ACR) provide guidance on suitable indications for commencing biologic therapy for RA recommending treatment for disease which is active despite optimization of conventional DMARDs or for patients with high disease activity and risk factors for poor outcome (ACPA and rheumatoid factor positivity and erosive disease on radiography) (Smolen et al., 2014).

As mentioned above, $\mathrm{TNF} \alpha$ is a key cytokine in the pathogenesis of synovial inflammation in RA, however, it has also been shown to play a vital role in fighting infections in animal models (Parks et al., 2011). TNF $\alpha$ is a type II transmembrane protein, cleaved by TNF- $\alpha$ converting enzyme (TACE) to a soluble form. It acts as a ligand for two receptors, TNFR1 and TNFR2, to enable transduction of anti-apoptotic, pro-inflammatory signals. TNF $\alpha$-mediated functions include phagosome maturation, autophagy inhibition and apoptosis following activation of caspase 8 by TNFR1 (Harris and Keane, 2010). By inhibiting these actions, anti-TNF therapy causes suppression of the immune system (Tracey et al., 2008) and improves outcomes for suppressing inflammation and function clinically. However, TNF inhibitor therapy has also been shown to induce neurological events (Kaltsonoudis et al., 2014).

\section{TYPES OF INFECTIONS}

The risk of developing infections is heightened in RA. In the following sections we describe the more common infections observed in people with RA. Since TNF inhibitor therapy can increase the incidence of infections in RA, it is possible that 
increased infections observed in RA during TNF inhibitor therapy could be a cause of RA flares, which in turn could have an impact on RA disease activity.

\section{BACTERIAL INFECTIONS}

Bacterial infections contribute to three quarters of infections in patients taking corticosteroids (CS), DMARDs or biological agents for RA and spondyloarthritis with respiratory tract infections being the most common (Germano et al., 2014). A recent systematic review of over 23,000 patients using anti-TNF therapy in the treatment of RA, juvenile idiopathic arthritis, ankylosing spondylitis, psoriatic arthritis, psoriasis and Crohn's disease, has further characterized infections associated with biologics. It showed that infections leading to a cessation in treatment included pneumonia (6.6\%), bacterial arthritis $(2.8 \%)$, gastrointestinal abscess $(2.1 \%)$, and cellulitis (1.1\%). In this study the highest rates of serious infection events were seen in RA and, within this subset of patients, cellulitis and pneumonia were the most common types of infection (Burmester et al., 2013).

\section{MYCOBACTERIAL INFECTIONS}

$\mathrm{TNF} \alpha$ is a key mediator in the host immune defense against mycobacterium tubercule bacillus (TB) infections leading to activation of macrophages, cell recruitment, granuloma formation and maintenance (Bean et al., 1999). In murine models, the neutralization of $\mathrm{TNF} \alpha$ increases susceptibility to primary $\mathrm{TB}$ and injection of soluble $\mathrm{TNF} \alpha$ receptors can cause activation of TB in infected mice (Senaldi et al., 1996). TNF inhibitor therapy may also result in active tuberculosis in humans carrying a latent infection (Keane et al., 2001). A 3 year study by Tubach (Tubach et al., 2009) found 69 cases of tuberculosis (40 in RA) in patients treated with anti-TNF $\alpha$. Tuberculosis has been found to occur more frequently in patients taking anti-TNF $\alpha$ therapy than in the general population with a standardized incidence rate varying between 12 and 35 (Ramiro et al., 2014). There is data to suggest that the risk of tuberculosis is not constant for all anti-TNF $\alpha$ medications, with soluble $\mathrm{TNF} \alpha$ receptors resulting in a lower risk of infection than monoclonal antibodies (Tubach et al., 2009).

\section{VIRAL AND OPPORTUNISTIC INFECTIONS}

Some studies separate opportunistic infections from a broader definition of infections, serious infections, or serious infection events. In a study of 23, 458 patients on anti-TNF $\alpha$ therapy 20 patients (14 patients with RA) developed opportunistic infections (excluding TB or oral candidiasis) including oesophageal candidiasis, aspergillosis, candida sepsis, coccidiomycosis, cytomegalovirus, herpes zoster, and nocardiasis (Burmester et al., 2013).

Analysis of a French registry for opportunistic infections in patients taking biologic therapy for any condition found that 43 patients had opportunistic infections; 29 on infliximab, 10 on adalimumab and 4 on etanercept (Salmon-Ceron et al., 2011). Twenty-six of these were experienced by patients with RA and, of the total 43, 10 patients required intensive care and four died. When interpreting these findings it should be noted that patient information included in registry data is unlikely to be subject to the close scrutiny and follow-up afforded as those from clinical trials. Thus, systematic reviews of randomized controlled trials and open-label trials may provide more robust data.

The risk of herpes zoster infection is higher in those with autoimmune conditions than in the general population (Wolfe et al., 2006) and rates of the disease are significantly increased for those receiving anti-TNF therapy (and monoclonal antibodies in particular), with an increased rate with greater age and higher disease activity at baseline (Strangfeld et al., 2009). However, a more recent systematic review including three studies investigating skin infections in patients taking biologic therapies calculated an adjusted hazard ratio range of 1.0-1.7 for herpes zoster infection, reflecting no significant risk of the infection when taking biological DMARDs (Ramiro et al., 2014).

\section{RISK OF INFECTION}

Randomized controlled trials are mixed in their demonstration of an association between biologic therapy and infectious adverse events (Lipsky et al., 2000; Keystone et al., 2004; Wallis et al., 2004). It has been demonstrated that of the patients with RA who acquire serious infections whilst taking biologics, 32\% permanently discontinue therapy (Burmester et al., 2013) leading to significant compromise in therapeutic options for the treatment of the patient's arthritis.

The higher rate of infectious events may result from an increased risk of infection inherent to RA (Doran et al., 2002a) (though this appears to have reduced over the last 50 years (Ni Mhuircheartaigh et al., 2013), which is thought to be secondary to immune disturbance associated with disease pathogenesis and the use of immune-modulators to control the condition (Doran et al., 2002a,b). An inherent risk of infection makes establishing a causal link between biologic DMARDs and infections more difficult. For this reason systematic reviews and meta-analyses of clinical trials and registry data are useful tools to investigate the rare adverse events, by pooling data for large cohorts of patients.

Recent work has suggested that the blood brain barrier (BBB) may be compromised in RA with macrophages being recruited from the systemic circulation via intercellular adhesion molecule (ICAM) expression on the endothelium (Jacobs and Tavitian, 2012). Increased cerebrovascular permeability has been demonstrated in mice challenged with the collagen-induced arthritis (CIA) model (Nishioku et al., 2011). The same group have also proposed that the S100A4 protein mediates disruption of the BBB by increasing vascular permeability (Nishioku et al., 2011). Further evidence that the BBB is not as immunologically a privileged site as previously thought is supported by the recent observations in mice with systemic inflammation triggered by a peripheral LPS challenge show activation of interferon-stimulated genes (ISGs) and TNF $\alpha$ in the brain (Thomson et al., 2014), as illustrated in Figure 1. Chronic inflammatory diseases, including RA, are observationally associated with neuropsychiatric features such as depression, anxiety, pain and fatigue. It was thought that these features were simply secondary to the peripheral manifestations of the disease. However, the above findings suggest the BBB may be more porous in RA (Nishioku et al., 2011)and that intracerebral $\mathrm{TNF} \alpha$ activity has been demonstrated and (Thomson et al., 2014), thus, opens the possibility of a primary, cerebral 


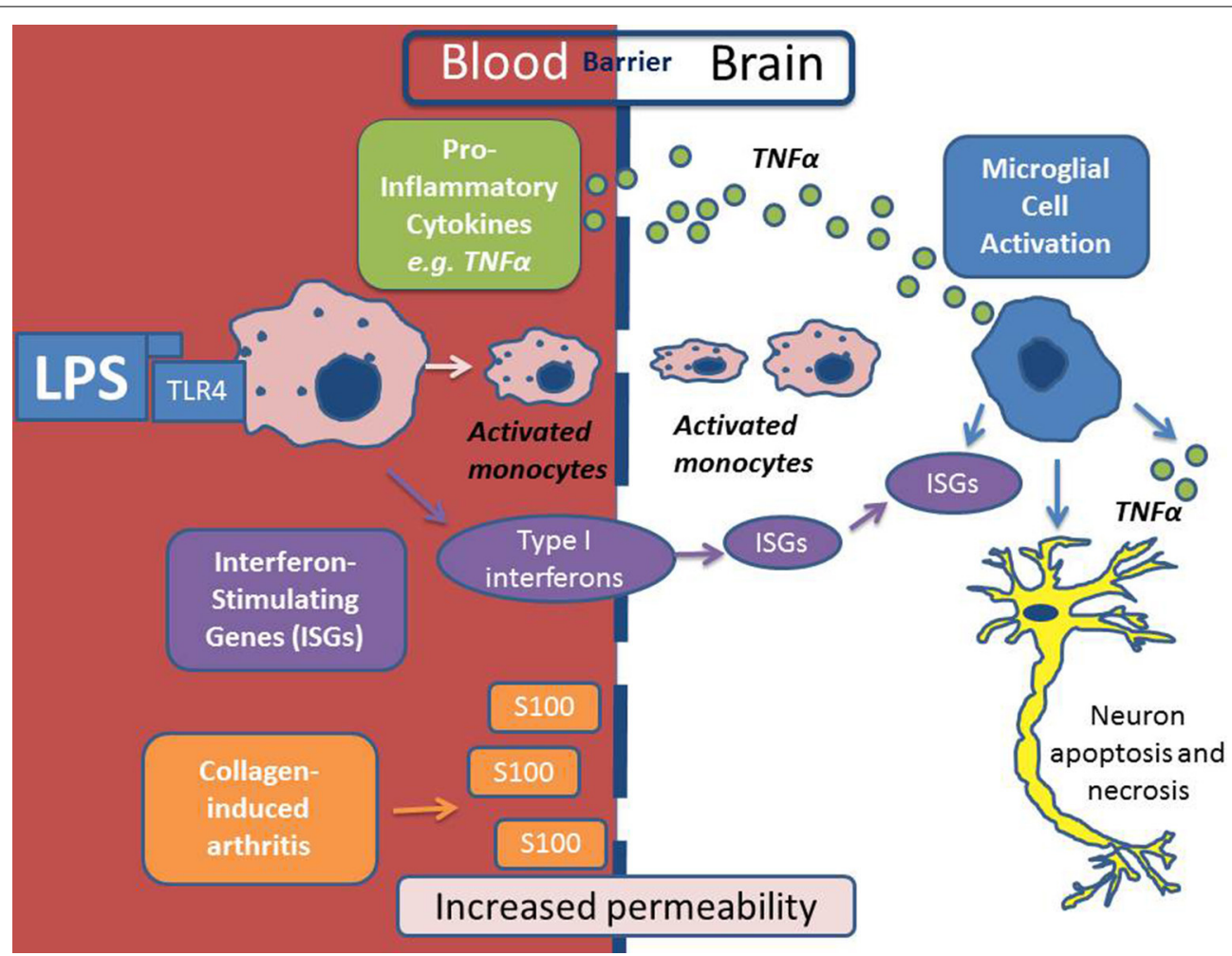

FIGURE 1 | Scheme depicting the mechanism of neuro-inflammation in autoimmune conditions. In this depiction lipopolysaccharide (LPS) is the inflammatory agent which causes activation of monocytes in the systemic circulation via Toll-Like Receptor 4 (TLR4). LPS/TLR4 activation results in the production of pro-inflammatory cytokines (including Tumor Necrosis Factor alpha (TNF $\alpha$ ) and Interferon Stimulating Genes (ISGs) which activate microglial cells within the brain. Activated monocytes are then recruited across the BBB and as a result of all of the above there may be consequential neuronal apoptosis and necrosis. In a model of collagen-induced arthritis, S100 was shown to increase BBB permeability to inflammatory cells. etiology for neuropsychiatric symptoms as proposed by Rech et al. (2013).

The influence of infections in triggering and then maintaining a pro-inflammatory response in RA is not fully understood. Laboratory-based studies have focused on the well-characterized pro-inflammatory response induced by agents such as LPS, which is clearly specific to gram negative bacteria. However, the risk of infection for patients on biologics for RA can also be influenced by patient characteristics including age, disease activity, leukopoenia, with co-morbidities including dementia, chronic lung disease, alcoholism and diabetes mellitus, baseline steroid use and female sex. An early systematic review of risk of serious infections in 5014 patients with RA taking anti-TNF therapy (infliximab and adalimumab with etanercept excluded due to differences in structure) in placebo-controlled trials found that serious infections requiring anti-microbial therapy or hospitalization occurred in 126 patients in the treatment arm and 26 patients on placebo (Bongartz et al., 2006). This demonstrated an increased risk of infection with an odds ratio of 2.0, which changed to 2.3 when stratified for dose (Bongartz et al., 2006). Similar findings have been found in other studies with a 2.7-2.8-fold increase in serious infections associated with infliximab and etanercept treatment (Listing et al., 2005) and an incidence rate of 1.79 with subcutaneous abatacept (Alten et al., 2014).
The most recent systematic review at the time of writing was an analysis of data to inform an update of EULAR recommendations for biologic therapies. This review concluded that most articles referring to serious infection demonstrated a significantly increased risk of infection in those treated with anti-TNF therapy when compared to conventional DMARD treatment (adjusted hazard ratio 1.0-1.7) (Ramiro et al., 2014).

A meta-analysis and simple pooling of data from 18 studies (8808 subjects) was used by Leombruno and colleagues to investigate the safety of TNF inhibitor therapy in RA (Leombruno et al., 2009). Etanercept was included in the analysis as the investigators considered that similarities out-weighed the differences between the agents. In this study there was no significant difference in the rate of serious infections between patients on anti-TNF treatment and those not. The mean duration of therapy was less than 1 year, therefore the authors recommended caution in drawing conclusions with regard to long-term safety of biologics. However, a later systematic review investigating the safety of further biologic treatment after initial anti-TNF failure found that serious infection rates were not significantly different to placebo (although only four trials met the eligibility criteria for the review) (Schoels et al., 2012), lending weight to the findings of Leombruno et al. (2009). A further meta-analysis of 12 randomized controlled trials investigating the risk of serious infections during rituximab, abatacept, and anakinra treatment for RA found no increased risk 
of infections for abatacept, rituximab or low-dose anakinra but an increased risk for high-dose anakinra (OR 3.4) (Salliot et al., 2009).

The risk of infection for patients on biologic therapy is affected by the other medications used to treat the condition. A study by Germano and colleagues demonstrated that, compared to the incidence ratio of infection in patients taking conventional DMARDS and corticosteroids, those taking anti-TNF and DMARDS had a two-fold increase in incidence ratio, and those taking anti-TNF and corticosteroids had a three-fold increase in incidence ratio (Germano et al., 2014). This dropped to a 2.5-fold increase with anti-TNF, corticosteroid and DMARD suggesting that corticosteroids increase the risk of infection when used with anti-TNF. Infection risk (though not serious infection risk) whilst taking adalimumab has been shown to increase with increasing dose of concurrent methotrexate therapy in biologic and DMARD naïve patients (Burmester et al., 2014). The risk of infections appears to vary depending on the biologic agent, with studies showing that opportunistic infections were less common when using etanercept compared to the monoclonal agents infliximab (OR 17.6) and adalimumab (OR 10.0) (Salmon-Ceron et al., 2011).

\section{TIMING OF INFECTIONS IN THE CONTEXT OF ANTI-TNF OR IMMUNOSUPPRESSANT TREATMENT}

Animal models have shown the peak of reported effects in the brain to occur within a few hours following the acute phase response (Serrats and Sawchenko, 2009). Peripheral LPS injection in mice has been shown to elicit Interferon Stimulating Genes (ISG) induction between 6 and $48 \mathrm{~h}$ after exposure (Thomson et al., 2014). However, Thomson and colleagues found that the ISG up regulation was not wholly TNF dependent, as shown by the lack of up regulation of all ISGs with TNF $\alpha$ alone (Thomson et al., 2014). The same group showed that IL-1beta and TNF alpha were both independently up regulated in the brain following multiple LPS challenges (Thomson et al., 2014). Nadeau and Rivest (1999) have also suggested that LPS can have rapid effects on the hypothalamic-pituitary-adrenal axis was determined by measuring the transcriptional activity of corticotropin-releasing factor and plasma corticosterone levels. It is therefore conceivable that clinical RA disease activity, which can flare after acute infection, may also be linked to neuro-inflammation during episodes of clinical infection.

Askling and Dixon (2008) found that infection risk was highest in the first few months of anti-TNF treatment, and then declined in frequency. This effect was echoed in another study demonstrating that the risk of serious infection was greater at 12 weeks duration of TNF inhibitor therapy (odds ratio 2.08) than at 104 weeks duration (odds ratio 0.97 ). This finding may be due to a shift in the drug safety profile of TNF inhibitor agents, with persistent blockade of the TNF pathway leading to up-regulation of an alternative immune pathway and thus a compensation of immunity (Rosenblum and Amital, 2011). However, the decline in infection risk with time may be due to confounding factors. As the active RA is controlled by the anti-TNF agent, the dose of immunosuppressant CS is reduced, leading to a possible recovery of host immunity. It is also hypothesized that some patients may be at an inherently higher risk of contracting infections. If they are included within the biologic treatment arm of a randomized controlled trial they may develop an infection soon after starting therapy, the drug will then be stopped and they will leave the cohort leaving a healthier cohort of patients. The latter is known as the "healthy user effect" (Rosenblum and Amital, 2011).

In terms of opportunistic infections (excluding TB), the median time from commencement of treatment to time of first opportunistic infection is 16.2 months (6.0-26.0) (Salmon-Ceron et al., 2011), much longer than the 3 months of highest risk, suggesting that immunosuppression secondary to biologics is, in fact, delayed in onset. It should be noted that studies since that performed by Dixon and colleagues (Askling and Dixon, 2008) have shown that the risk of first serious infection was stable throughout treatment.

Numerous murine and human models have demonstrated how LPS-induced gram-negative infections induce inflammation accompanied by somatic or visceral pain (Teeling et al., 2012; Chiu et al., 2013; Thomson et al., 2014). Such features have been attributed to sensitisation of nociceptors by inflammatory mediators released by immune cells. Sensitisation of nociceptors by inflammatory mediators released by peripheral immune cells occurs through activation of the TLR4 signaling pathway by LPS a toxic product of bacterial lysis. Meseguer et al. (2014) recently proposed that LPS induces activation of TRPA1 in a murine model, a transient receptor potential cation channel that trasduces environmental stimuli to nociceptor activity. The group also found that acute vascular reactions, including neurogenic inflammation as indicated by CGRP release (measured by enzyme immunoassay), were mediated through TRPA1 and were independent of TLR4 activation. Such observations suggest that not only are infectious agents such as LPS inducing the pain response, but also produce a neurovascular response which could explain the missing link between peripheral infection and central brain neuro-inflammation.

\section{NEURO-IMMUNE INTERACTION IN RHEUMATOID ARTHRITIS}

Neuroinflammation is conducted, in the CNS, by microglial cells and astrocytes through the production of pro-inflammatory mediators and cytokines. This inflammation results in cell death and neuronal loss. RA is characterized by chronic inflammation which leads to a constant stimulation of nociceptor and excitation of afferent neurons to the brain. As such it has neurological similarities to a chronic pain state and central sensitization is thought to occur in as a result (Woolf, 1983). Evidence for enhanced hyperalgesia has been demonstrated in RA patients when compared to controls using a capsaicin and pin-prick method (Morris et al., 1997).

Lee and colleagues have suggested central sensitization as a mechanism for explaining chronic pain in rheumatic diseases such as RA (Lee et al., 2011). Central sensitization and changes in nociceptive processing in response to repeated stimulation by chemo-somatosensory event-related potentials (CSSERP) have been demonstrated encephalographically in RA patients (Wendler et al., 2001). Although increases in alpha 1 and beta 
range are thought to be secondary to a chronic pain state it may be that these changes are in fact a result of direct neuroinflammation secondary to the arthritis itself. Schweinhardt et al. found that people with painful RA demonstrate higher activation of brain regions associated with emotional cognition of pain including the medial pre-frontal cortex (Schweinhardt et al., 2008).

Further evidence of neuro-immune interplay has also emerged suggesting that use of biologic therapies such as TNF inhibitors can have central brain effects in people with RA well before a reduction of inflammation within affected joints occurs. In a recent study functional MRI (fMRI) was used to assess the neurological effects of TNF inhibitor therapy in 10 patients with active RA (Rech et al., 2013). The fMRI signal is sensitive to concentration of deoxyhemoglobin (which is paramagnetic), hence can detect areas of higher neuronal activity where there is greater blood flow due to increased brain metabolism associated with a task or stimulus (Ogawa et al., 1990). Rech et al. (2013) analyzed the fMRI response to compression of finger joints in RA patients at baseline and 3, 7, and 28 days after subcutaneous treatment with the TNF inhibitor adalimumab. Clinical disease activity was assessed at the same time points by applying the validated DAS28 (Disease Activity Score), which is based on the assessment of the number of swollen and tender joints, the ESR and the patient's global rating of disease activity. Inflammatory tissue was also measured anatomically by MRI of the dominantly affected hand at baseline and 28 days. At baseline there was a greater volume of functional activation in response to finger joint compression of the patients that responded to TNF treatment than those that did not respond. The main regions activated by joint compression at baseline were the somatosensory cortex, insular cortex and dorsolateral prefrontal cortex, thus suggesting a greater perception of pain. However, the brain activation was then significantly reduced as early as 3 days after treatment and further decreased after 7 days, whereas the disease activity score was only significantly reduced by day 28. Data from this study support the concept that chronic inflammation in RA leads to central sensitization, with the possibility of modulating pain perception with appropriate treatment.

A neuro-immune interaction is not isolated to RA or TNF $\alpha$. Neuropsychiatric symptoms have shown improvement with cytokine blockade, for example IL-1 inhibition in Sjögrens and diabetes mellitus reduced fatigue (Norheim et al., 2012) Within "pure" neuroinflammatory disorders such as Alzheimer's there is increasing murine data to show an improvement in memory and reduced hippocampal TNF $\alpha$ levels as a result of anti-TNF $\alpha$ receptor fusion protein administration (Detrait et al., 2014). This has lead to a move toward considering anti-TNF $\alpha$ as a therapy for Alzheimer's dementia (Cheng et al., 2014) Auanofin, a gold-containing medication and established treatment for RA, dampens inflammation through manipulation of the antiand pro-inflammatory interleukin balance. Interestingly a recent in vitro study has demonstrated a reduced production of cytotoxic mediators by microglia in response to gold therapy (Medeiros et al., 2007).

Diamond and Tracey recently proposed the concept of the immunological homunculus of the brain (Diamond and Tracey, 2011) where the brain acts as a sensory organ, allowing real-time transmission of information such as infections, tissue damage and inflammation to the central nervous system. Indeed, they draw attention to the concept of an "inflammatory reflex," in which the neurotransmitter acetyl choline interacts with cytokine production via $\alpha 7$ nicotinic acetylcholine receptors on immune cells.

\section{MAGNETIC RESONANCE IMAGING IN RHEUMATOID ARTHRITIS}

Magnetic resonance imaging (MRI) provides a variety of techniques for assessing neurological involvement of disease processes by detecting changes in brain morphometry [T1 weighted imaging $(\mathrm{T} 1 \mathrm{w})]$ and tissue microstructure [T2 weighted imaging (T2w), diffusion weighted imaging (DWI), diffusion tensor imaging (DTI), and magnetisation transfer imaging (MTI)], brain metabolism by magnetic resonance spectroscopy (MRS) and actual brain functionality by fMRI as described above. However, there are far fewer reports of neuroimaging abnormalities for RA compared to other related autoimmune diseases. In Sjögren's syndrome significant loss of brain tissue and presence of white matter lesions are observed (Akasbi et al., 2012; Lauvsnes et al., 2014).Lupus can lead to microstructural changes in white matter (WM), white matter lesions and metabolic abnormalities (Axford et al., 2001; Zivadinov et al., 2013) with associated neuropsychological sequelae. A summary of the imaging techniques utilized so far in demonstrating inflammatory changes in autoimmunemediated conditions is summarized in Table 1.

T2 weighted imaging allows detection of lesions associated with inflammatory and degenerative changes in the brain and in terms of number and size of WM hyperintensities, lesion load is higher with age in the normal population and associated with microvascular disease (King et al., 2014). A quantitative T2w MRI study showed no significant difference in WM lesion load between RA patients and controls (Bekkelund et al., 1995) although WM lesions in RA patients have been associated with higher levels of the protein S100B, a potential plasma marker of $\mathrm{BBB}$ disruption and neurodegenerative effects (Hamed et al., 2012). Hamed and colleagues performed cognitive testing and found that RA patients had greater depression than controls, but this did not correlate with cognitive scores, whereas cognition did correlate with S100B levels (Hamed et al., 2012). In a rodent model of arthritis the presence of the inflammation stimulated protein S100A4 was strongly associated with BBB disruption (Nishioku et al., 2011). Thus a cerebrovascular component is likely in RA, but not one specifically detectable by conventional MRI in the general RA population.

T1 weighted imaging provides high spatial resolution of the brain with excellent contrast between gray and white matter structures to allow assessment of the shape and size of different anatomical regions. A recent study with T1w MRI demonstrated no global difference of intracranial volume between RA patients and controls, but observed significant increases in the volume of basal ganglia structures (Wartolowska et al., 2012). The caudate nucleus in particular showed the most significant increase, suggesting these changes are related to pain processing rather than the disease itself. However, there are reports of both increased and decreased gray matter structure volumes in patients with chronic 
Table 1 | A table displaying the imaging modalities used to investigate neuroinflammation.

\begin{tabular}{lll}
\hline Imaging modality & Method of action & Relevance to rheumatoid arthritis \\
\hline T2-Weighted MRI & $\begin{array}{l}\text { Inflammatory and degenerative changes are } \\
\text { demonstrated in the form of white matter } \\
\text { hyperintensities }\end{array}$ & $\begin{array}{l}\text { White matter lesions are associated with higher levels of S100B } \\
\text { which is a potential surrogate marker for blood brain barrier } \\
\text { disruption (Hamed et al., 2012) }\end{array}$ \\
\hline T1-Weighted MRI & $\begin{array}{l}\text { Provides high spatial resolution imaging of the brain } \\
\text { allowing volumetric assessment of cerebral anatomy }\end{array}$ & $\begin{array}{l}\text { Has demonstrated increased volume of basal ganglia in RA } \\
\text { patients compared to controls (Steens et al., 2005) }\end{array}$ \\
\hline $\begin{array}{l}\text { Diffusion weighted } \\
\text { imaging }\end{array}$ & $\begin{array}{l}\text { Quantitative parameters relate to neuronal density, } \\
\text { structural integrity and water content }\end{array}$ & $\begin{array}{l}\text { Potential for assessing inflammatory and neurodegenerative } \\
\text { changes (Steens et al., 2005; Zivadinov et al., 2013) }\end{array}$ \\
\hline $\begin{array}{l}\text { Magnetisation } \\
\text { transfer imaging }\end{array}$ & $\begin{array}{l}\text { Quantitative parameters relate to magnetisation } \\
\text { exchange between macromolecules (e.g., myelin, } \\
\text { proteins) and bulk tissue water }\end{array}$ & $\begin{array}{l}\text { Potential for assessing inflammatory and neurodegenerative } \\
\text { changes (Steens et al., 2005) }\end{array}$ \\
\hline $\begin{array}{l}\text { Magnetic resonance } \\
\text { spectroscopy }\end{array}$ & $\begin{array}{l}\text { Relative levels of specific metabolites, including N-acetyl } \\
\text { aspartate, creatine, choline, myo-Inositol and some } \\
\text { neurotransmitters }\end{array}$ & $\begin{array}{l}\text { Increased choline/creatine ratio is proportional to rise in ESR and } \\
\text { disease activity } \\
\text { Increased choline levels seen in neuroinflammation in systemic } \\
\text { lupus (Axford et al., 2001; Emmer et al., 2009) }\end{array}$ \\
\hline
\end{tabular}

pain indicating there may be a complex mix of neurodegenerative or adaptive changes dependent on disease, duration and consequent lifestyle changes, as well as treatment (Wartolowska et al., 2012). Currently it is known that only in patients with longstanding (i.e., $>15$ years) RA is there a reduction in brain volume that may relate to neurodegenerative changes caused by RA (Bekkelund et al., 1995).

Treatment for RA commonly includes CS to reduce inflammation and the effect of low-dose CS treatment on the brain has been investigated with quantitative MRI (Steens et al., 2005). Parameters derived from DWI and MTI relate to neuronal density and myelin-tissue water magnetisation exchange respectively, hence are sensitive to inflammatory and neurodegenerative changes. Whole brain histograms of the apparent diffusion coefficient (ADC) and magnetization transfer ratio (MTR) showed no significant difference between controls and RA patients with or without CS treatment. Although the results of Steens et al. (2005) indicate no global differences of MRI parameters, a more focused study that separates gray and white matter structures is warranted as performed for lupus and with the increased sensitivity of modern 3T MRI systems (Zivadinov et al., 2013). In addition, DTI has much greater sensitivity than DWI to detect microstructural changes in WM, and can provide detailed anatomical maps that describe regional differences of neurodegenerative change (Barrick et al., 2010).

There is some evidence of metabolic changes in the brain due to RA. In the Steens study (Steens et al., 2005) 1H MRS of a single white matter region adjacent to the left ventricle almost reached a significant difference in metabolite levels between RA patients and controls. A subsequent retrospective analysis showed that a high choline to creatine $(\mathrm{Cho} / \mathrm{Cr})$ metabolite ratio was associated with high ESR levels and correlated with ESR and disease activity after correction for disease activity and duration (Emmer et al., 2009). There was no difference in $\mathrm{N}$-acetyl aspartate to creatine (NAA/Cr) ratio or correlation with ESR, suggesting there was no neuronal damage, which is consistent with the lack of major neurological symptoms in RA, unlike for lupus. Elevated choline is found in lupus and other neuroinflammatory diseases (Axford et al., 2001). Choline has a function in cell membrane synthesis and its elevation in RA may relate to microglial activation or monocyte infiltration (Emmer et al., 2009). Brain metabolic changes have been associated with pain processing regions in a number of studies and elevation of the combined neurotransmitters glutamate and glutamine observed in fibromyalgia patients (Fayed et al., 2010) and also with induced pain (Gussew et al., 2010). The significance of these results for development of novel treatment or monitoring treatment response is yet to be established.

To date there are far fewer neuroimaging studies in RA patients compared to other diseases with cerebrovascular, neuroinflammatory and neurodegenerative components (e.g., stroke, lupus and dementias). This reflects the lower incidence of neurological symptoms and the more subtle effects of cerebrovascular involvement that have been so far detected.

\section{CONCLUSION}

The concept of neuroinflammation as a significant component of disease pathophysiology in RA has only recently been recognized. Animal models have recently demonstrated that infectious agents including LPS can directly activate the immune system stimulate pain responses. Coupled with the strong link between RA and incidence of infection, it may be time to reconsider the balance between maintaining disease remission using disease-modifying immune-modulatory drugs and preventing disease flares induced by infection. However, the link between infection and immunemodulatory therapies remains controversial and comparison of studies is made difficult by differing biologic therapies used, different diseases treated and different, concurrent medications regimes. Further, high-powered studies are required to establish the period of highest risk and susceptibility to infections and the overall risk of infections for patients taking biological treatments for RA. 
Future work is needed to understand how the RA disease process impacts on pain activation in RA, whether sensitisation is a general phenomenon in RA or found in specific subsets of patients and what specific infectious triggers contribute to an autoimmune pro-inflammatory network that leads to chronic pain and inflammation.

\section{REFERENCES}

Akasbi, M., Berenguer, J., Saiz, A., Brito-Zeron, P., Perez-De-Lis, M., Bove, A., et al. (2012). White matter abnormalities in primary Sjogren syndrome. QJM 105, 433-443. doi: 10.1093/qjmed/hcr218

Alten, R., Kaine, J., Keystone, E., Nash, P., Delaet, I., and Genovese, M. C. (2014). Long-term safety of subcutaneous abatacept in rheumatoid arthritis: integrated analysis of clinical trial data of up to 4.75 years of treatment. Arthritis Rheumatol. 66, 1987-1997. doi: 10.1002/art.38687

Askling, J., and Dixon, W. (2008). The safety of anti-tumour necrosis factor therapy in rheumatoid arthritis. Curr. Opin. Rheumatol. 20, 138-144. doi: 10.1097/BOR.0b013e3282f4b392

Axford, J. S., Howe, F. A., Heron, C., and Griffiths, J. R. (2001). Sensitivity of quantitative (1) H magnetic resonance spectroscopy of the brain in detecting early neuronal damage in systemic lupus erythematosus. Ann. Rheum. Dis. 60, 106-111. doi: 10.1136/ard.60.2.106

Barrick, T. R., Charlton, R. A., Clark, C. A., and Markus, H. S. (2010). White matter structural decline in normal ageing: a prospective longitudinal study using tract-based spatial statistics. Neuroimage 51, 565-577. doi: 10.1016/j.neuroimage.2010.02.033

Bean, A. G., Roach, D. R., Briscoe, H., France, M. P., Korner, H., Sedgwick, J. D., et al. (1999). Structural deficiencies in granuloma formation in TNF gene-targeted mice underlie the heightened susceptibility to aerosol Mycobacterium tuberculosis infection, which is not compensated for by lymphotoxin. J. Immunol. 162, 3504-3511.

Bekkelund, S. I., Pierre-Jerome, C., Husby, G., and Mellgren, S. I. (1995). Quantitative cerebral MR in rheumatoid arthritis. AJNR Am. J. Neuroradiol. 16, 767-772.

Bongartz, T., Sutton, A. J., Sweeting, M. J., Buchan, I., Matteson, E. L., and Montori, V. (2006). Anti-TNF antibody therapy in rheumatoid arthritis and the risk of serious infections and malignancies: systematic review and meta-analysis of rare harmful effects in randomized controlled trials. JAMA 295, 2275-2285. doi: 10.1001/jama.295.19.2275

Burmester, G. R., Kivitz, A. J., Kupper, H., Arulmani, U., Florentinus, S., Goss, S. L., et al. (2014). Efficacy and safety of ascending methotrexate dose in combination with adalimumab: the randomised CONCERTO trial. Ann. Rheum. Dis. doi: 10.1136/annrheumdis-2013-204769. [Epub ahead of print].

Burmester, G. R., Panaccione, R., Gordon, K. B., McIlraith, M. J., and Lacerda, A. P. (2013). Adalimumab: long-term safety in 23458 patients from global clinical trials in rheumatoid arthritis, juvenile idiopathic arthritis, ankylosing spondylitis, psoriatic arthritis, psoriasis and Crohn's disease. Ann. Rheum. Dis. 72, 517-524. doi: 10.1136/annrheumdis-2011-201244

Capellino, S., Cosentino, M., Wolff, C., Schmidt, M., Grifka, J., and Straub, R. H. (2010). Catecholamine-producing cells in the synovial tissue during arthritis: modulation of sympathetic neurotransmitters as new therapeutic target. Ann. Rheum. Dis. 69, 1853-1860. doi: 10.1136/ard.2009. 119701

Cheng, X., Shen, Y., and Li, R. (2014). Targeting TNF: a therapeutic strategy for Alzheimer's disease. Drug Discov. Today. doi: 10.1016/j.drudis.2014. 06.029. [Epub ahead of print].

Chiu, I. M., Heesters, B. A., Ghasemlou, N., Von Hehn, C. A., Zhao, F., Tran, J., et al. (2013). Bacteria activate sensory neurons that modulate pain and inflammation. Nature 501, 52-57. doi: 10.1038/nature12479

Chou, R., Kane, M., Ghimire, S., and Gautam, S. (2010). Tumor Necrosis Factor Inhibition Reduces the Incidence of Alzheimer's Disease in Rheumatoid Arthritis Patients, Atlanta, GA: American College of Rheumatology.

Combs, C. K., Karlo, J. C., Kao, S. C., and Landreth, G. E. (2001). Beta-Amyloid stimulation of microglia and monocytes results in TNFalpha-dependent expression of inducible nitric oxide synthase and neuronal apoptosis. J. Neurosci. 21, 1179-1188.

De Almeida, D. E., Ling, S., Pi, X., Hartmann-Scruggs, A. M., Pumpens, P., and Holoshitz, J. (2010). Immune dysregulation by the rheumatoid arthritis shared epitope. J. Immunol. 185, 1927-1934. doi: 10.4049/jimmunol.09 04002

De Rycke, L., Peene, I., Hoffman, I. E., Kruithof, E., Union, A., Meheus, L., et al. (2004). Rheumatoid factor and anticitrullinated protein antibodies in rheumatoid arthritis: diagnostic value, associations with radiological progression rate, and extra-articular manifestations. Ann. Rheum. Dis. 63, 1587-1593. doi: 10.1136/ard.2003.017574

Detrait, E. R., Danis, B., Lamberty, Y., and Foerch, P. (2014). Peripheral administration of an anti-TNF-alpha receptor fusion protein counteracts the amyloid induced elevation of hippocampal TNF-alpha levels and memory deficits in mice. Neurochem. Int. 72, 10-13. doi: 10.1016/j.neuint.2014.04.001

Diamond, B., and Tracey, K. J. (2011). Mapping the immunological homunculus. Proc. Natl. Acad. Sci. U.S.A. 108, 3461-3462. doi: 10.1073/pnas.1100329108

Doran, M. F., Crowson, C. S., Pond, G. R., O'Fallon, W. M., and Gabriel, S. E. (2002a). Frequency of infection in patients with rheumatoid arthritis compared with controls: a population-based study. Arthritis Rheum. 46, 2287-2293. doi: 10.1002/art.10524

Doran, M. F., Crowson, C. S., Pond, G. R., O’Fallon, W. M., and Gabriel, S. E. (2002b). Predictors of infection in rheumatoid arthritis. Arthritis Rheum. 46, 2294-2300. doi: 10.1002/art.10529

Emmer, B. J., van der Bijl, A. E., Huizinga, T. W., Breedveld, F. C., Steens, S. C., Th Bosma, G. P., et al. (2009). Brain involvement in rheumatoid arthritis: a magnetic resonance spectroscopy study. Arthritis Rheum. 60, 3190-3195. doi: 10.1002/art.24932

Fayed, N., Garcia-Campayo, J., Magallon, R., Andres-Bergareche, H., Luciano, J. V., Andres, E., et al. (2010). Localized 1H-NMR spectroscopy in patients with fibromyalgia: a controlled study of changes in cerebral glutamate/glutamine, inositol, choline, and N-acetylaspartate. Arthritis Res. Ther. 12, R134. doi: 10.1186/ar3072

Feldmann, M., and Maini, R. N. (2010). Anti-TNF therapy, from rationale to standard of care: what lessons has it taught us? J. Immunol. 185, 791-794. doi: 10.4049/jimmunol.1090051

Firestein, G. S. (2003). Evolving concepts of rheumatoid arthritis. Nature 423, 356-361. doi: 10.1038/nature01661

Germano, V., Cattaruzza, M. S., Osborn, J., Tarantino, A., Di Rosa, R., Salemi, S., et al. (2014). Infection risk in rheumatoid arthritis and spondyloarthropathy patients under treatment with DMARDs, corticosteroids and TNF-alpha antagonists. J. Transl. Med. 12:77. doi: 10.1186/1479-5876-12-77

Gregersen, P. K., Silver, J., and Winchester, R. J. (1987). The shared epitope hypothesis. An approach to understanding the molecular genetics of susceptibility to rheumatoid arthritis. Arthritis Rheum. 30, 1205-1213. doi: 10.1002/art.1780301102

Gussew, A., Rzanny, R., Erdtel, M., Scholle, H. C., Kaiser, W. A., Mentzel, H. J., et al. (2010). Time-resolved functional 1H MR spectroscopic detection of glutamate concentration changes in the brain during acute heat pain stimulation. Neuroimage 49, 1895-1902 doi: 10.1016/j.neuroimage.2009.09.007

Hamed, S. A., Selim, Z. I., Elattar, A. M., Elserogy, Y. M., Ahmed, E. A., and Mohamed, H. O. (2012). Assessment of biocorrelates for brain involvement in female patients with rheumatoid arthritis. Clin. Rheumatol. 31, 123-132. doi: 10.1007/s10067-011-1795-1

Harris, J., and Keane, J. (2010). How tumour necrosis factor blockers interfere with tuberculosis immunity. Clin. Exp. Immunol. 161, 1-9. doi: 10.1111/j.13652249.2010.04146.x

Ho, L., Purohit, D., Haroutunian, V., Luterman, J. D., Willis, F., Naslund, J., et al. (2001). Neuronal cyclooxygenase 2 expression in the hippocampal formation as a function of the clinical progression of Alzheimer disease. Arch. Neurol. 58, 487-492. doi: 10.1001/archneur.58.3.487

Hull, M., Muksch, B., Akundi, R. S., Waschbisch, A., Hoozemans, J. J., Veerhuis, R., et al. (2006). Amyloid beta peptide (25-35) activates protein kinase C leading to cyclooxygenase- 2 induction and prostaglandin E2 release in primary midbrain astrocytes. Neurochem. Int. 48, 663-672. doi: 10.1016/j.neuint.2005.08.013

Hunter, R. L., Dragicevic, N., Seifert, K., Choi, D. Y., Liu, M., Kim, H. C., et al. (2007). Inflammation induces mitochondrial dysfunction and dopaminergic neurodegeneration in the nigrostriatal system. J. Neurochem. 100, 1375-1386. doi: 10.1111/j.1471-4159.2006.04327.x

Inan, A. S., Masatlioglu, S., Ozyurek, S. C., Engin, D., and Erdem, I. (2011). Unusual central nervous system involvement of rheumatoid arthritis: successful treatment with steroid and azathioprine. Rheumatol. Int. 31, 1383-1385. doi: 10.1007/s00296-009-1266-z 
Jacobs, A. H., and Tavitian, B. (2012). INMiND consortium. Noninvasive molecular imaging of neuroinflammation. J. Cereb. Blood Flow Metab. 32, 1393-1415. doi: $10.1038 /$ jcbfm.2012.53

Kaltsonoudis, E., Zikou, A. K., Voulgari, P. V., Konitsiotis, S., Argyropoulou, M. I., and Drosos, A. A. (2014). Neurological adverse events in patients receiving antiTNF therapy: a prospective imaging and electrophysiological study. Arthritis Res. Ther. 16, R125. doi: 10.1186/ar4582

Keane, J., Gershon, S., Wise, R. P., Mirabile-Levens, E., Kasznica, J., Schwieterman, W. D., et al. (2001). Tuberculosis associated with infliximab, a tumor necrosis factor alpha-neutralizing agent. N. Engl. J. Med. 345, 1098-1104. doi: 10.1056/NEJMoa011110

Keystone, E. C., Kavanaugh, A. F., Sharp, J. T., Tannenbaum, H., Hua, Y., Teoh, L. S., et al. (2004). Radiographic, clinical, and functional outcomes of treatment with adalimumab (a human anti-tumor necrosis factor monoclonal antibody) in patients with active rheumatoid arthritis receiving concomitant methotrexate therapy: a randomized, placebo-controlled, 52-week trial. Arthritis Rheum. 50, 1400-1411. doi: 10.1002/art.20217

King, K. S., Peshock, R. M., Rossetti, H. C., McColl, R. W., Ayers, C. R., Hulsey, K. M., et al. (2014). Effect of normal aging versus hypertension, abnormal body mass index, and diabetes mellitus on white matter hyperintensity volume. Stroke 45, 255-257. doi: 10.1161/STROKEAHA.113.003602

Klareskog, L., Ronnelid, J., Lundberg, K., Padyukov, L., and Alfredsson, L. (2008). Immunity to citrullinated proteins in rheumatoid arthritis. Annu. Rev. Immunol. 26, 651-675. doi: 10.1146/annurev.immunol.26.021607. 090244

Lauvsnes, M. B., Beyer, M. K., Appenzeller, S., Greve, O. J., Harboe, E., Goransson, L. G., et al. (2014). Loss of cerebral white matter in primary Sjogren's syndrome: a controlled volumetric magnetic resonance imaging study. Eur. J. Neurol. 21, 1324-1329. doi: 10.1111/ene. 12486

Lee, Y. C., Nassikas, N. J., and Clauw, D. J. (2011). The role of the central nervous system in the generation and maintenance of chronic pain in rheumatoid arthritis, osteoarthritis and fibromyalgia. Arthritis Res. Ther. 13, 211. doi: 10.1186/ar3306

Leombruno, J. P., Einarson, T. R., and Keystone, E. C. (2009). The safety of anti-tumour necrosis factor treatments in rheumatoid arthritis: meta and exposure-adjusted pooled analyses of serious adverse events. Ann. Rheum. Dis. 68, 1136-1145. doi: 10.1136/ard.2008.091025

Lipsky, P. E., van der Heijde, D. M., St. Clair, E. W., Furst, D. E., Breedveld, F. C., Kalden, J. R., et al. (2000). Infliximab and methotrexate in the treatment of rheumatoid arthritis. Anti-Tumor Necrosis Factor Trial in Rheumatoid Arthritis with Concomitant Therapy Study Group. N. Engl. J. Med. 343, 1594-1602. doi: 10.1056/NEJM200011303432202

Listing, J., Strangfeld, A., Kary, S., Rau, R., von Hinueber, U., Stoyanova-Scholz, M., et al. (2005). Infections in patients with rheumatoid arthritis treated with biologic agents. Arthritis Rheum. 52, 3403-3412. doi: 10.1002/art.21386

MacGregor, A. J., Snieder, H., Rigby, A. S., Koskenvuo, M., Kaprio, J., Aho, K., et al. (2000). Characterizing the quantitative genetic contribution to rheumatoid arthritis using data from twins. Arthritis Rheum. 43, 30-37. doi: 10.1002/15290131(200001)43:1<30::AID-ANR5>3.0.CO;2-B

Mahdi, H., Fisher, B. A., Kallberg, H., Plant, D., Malmstrom, V., Ronnelid, J., et al. (2009). Specific interaction between genotype, smoking and autoimmunity to citrullinated alpha-enolase in the etiology of rheumatoid arthritis. Nat. Genet. 41, 1319-1324. doi: 10.1038/ng.480

Malaviya, A. P., and Ostor, A. J. (2012). Rheumatoid arthritis and the era of biologic therapy. Inflammopharmacology 20, 59-69. doi: 10.1007/s10787-012-0123-y

Markenson, J. A., McDougal, J. S., Tsairis, P., Lockshin, M. D., and Christian, C. L. (1979). Rheumatoid meningitis: a localized immune process. Ann. Intern. Med. 90, 786-789. doi: 10.7326/0003-4819-90-5-786

McInnes, I. B., and Schett, G. (2011). The pathogenesis of rheumatoid arthritis. N. Engl. J. Med. 365, 2205-2219. doi: 10.1056/NEJMra1004965

Medeiros, R., Prediger, R. D., Passos, G. F., Pandolfo, P., Duarte, F. S., Franco, J. L., et al. (2007). Connecting TNF-alpha signaling pathways to iNOS expression in a mouse model of Alzheimer's disease: relevance for the behavioral and synaptic deficits induced by amyloid beta protein. J. Neurosci. 27, 5394-5404. doi: 10.1523/JNEUROSCI.5047-06.2007

Mellor, R. (1959). Cellular origin of rheumatoid factor. J. Exp. Med. 110, 875-876. doi: 10.1084/jem.110.6.875

Meseguer, V., Alpizar, Y. A., Luis, E., Tajada, S., Denlinger, B., Fajardo, O., et al. (2014). TRPAl channels mediate acute neurogenic inflammation and pain produced by bacterial endotoxins. Nat. Commun. 5, 3125. doi: 10.1038/ncomms4125

Mikuls, T. R., Payne, J. B., Yu, F., Thiele, G. M., Reynolds, R. J., Cannon, G. W., et al. (2014). Periodontitis and Porphyromonas gingivalis in patients with rheumatoid arthritis. Arthritis Rheumatol. 66, 1090-1100. doi: 10.1002/art.38348

Morris, V. H., Cruwys, S. C., and Kidd, B. L. (1997). Characterisation of capsaicininduced mechanical hyperalgesia as a marker for altered nociceptive processing in patients with rheumatoid arthritis. Pain 71, 179-186. doi: 10.1016/S03043959(97)03361-7

Nadeau, S., and Rivest, S. (1999). Effects of circulating tumor necrosis factor on the neuronal activity and expression of the genes encoding the tumor necrosis factor receptors (p55 and p75) in the rat brain: a view from the blood-brain barrier. Neuroscience 93, 1449-1464. doi: 10.1016/S0306-4522(99)00225-0

Ni Mhuircheartaigh, O. M., Matteson, E. L., Green, A. B., and Crowson, C. S. (2013). Trends in serious infections in rheumatoid arthritis. J. Rheumatol. 40, 611-616. doi: 10.3899/jrheum.121075

Nishioku, T., Furusho, K., Tomita, A., Ohishi, H., Dohgu, S., Shuto, H., et al. (2011). Potential role for S100A4 in the disruption of the blood-brain barrier in collagen-induced arthritic mice, an animal model of rheumatoid arthritis. Neuroscience 189, 286-292. doi: 10.1016/j.neuroscience.2011.05.044

Norheim, K. B., Harboe, E., Goransson, L. G., and Omdal, R. (2012). Interleukin-1 inhibition and fatigue in primary Sjogren's syndrome-a double blind, randomised clinical trial. PLoS ONE 7:e30123. doi: 10.1371/journal.pone.0030123

O'Callaghan, J. P., Sriram, K., and Miller, D. B. (2008). Defining "neuroinflammation." Ann. N.Y. Acad. Sci. 1139, 318-330. doi: 10.1196/annals.1432.032

Ogawa, S., Lee, T. M., Kay, A. R., and Tank, D. W. (1990). Brain magnetic resonance imaging with contrast dependent on blood oxygenation. Proc. Natl. Acad. Sci. U.S.A. 87, 9868-9872. doi: 10.1073/pnas.87.24.9868

Panayi, G. S. (2006). Even though T-cell-directed trials have been of limited success, is there reason for optimism? Nat. Clin. Pract. Rheumatol. 2, 58-59. doi: 10.1038/ncprheum0094

Parks, E. L., Geha, P. Y., Baliki, M. N., Katz, J., Schnitzer, T. J., and Apkarian, A. V. (2011). Brain activity for chronic knee osteoarthritis: dissociating evoked pain from spontaneous pain. Eur. J. Pain 15, 843.e1-843.e14. doi: 10.1016/j.ejpain.2010.12.007

Ramiro, S., Gaujoux-Viala, C., Nam, J. L., Smolen, J. S., Buch, M., Gossec, L., et al. (2014). Safety of synthetic and biological DMARDs: a systematic literature review informing the 2013 update of the EULAR recommendations for management of rheumatoid arthritis. Ann. Rheum. Dis. 73, 529-535. doi: 10.1136/annrheumdis-2013-204575

Rech, J., Hess, A., Finzel, S., Kreitz, S., Sergeeva, M., Englbrecht, M., et al. (2013). Association of brain functional magnetic resonance activity with response to tumor necrosis factor inhibition in rheumatoid arthritis. Arthritis Rheum. 65, 325-333. doi: 10.1002/art.37761

Rosenblum, H., and Amital, H. (2011). Anti-TNF therapy: safety aspects of taking the risk. Autoimmun. Rev. 10, 563-568. doi: 10.1016/j.autrev.2011.04.010

Salliot, C., Dougados, M., and Gossec, L. (2009). Risk of serious infections during rituximab, abatacept and anakinra treatments for rheumatoid arthritis: metaanalyses of randomised placebo-controlled trials. Ann. Rheum. Dis. 68, 25-32. doi: 10.1136/ard.2007.083188

Salmon-Ceron, D., Tubach, F., Lortholary, O., Chosidow, O., Bretagne, S., Nicolas, N., et al. (2011). Drug-specific risk of non-tuberculosis opportunistic infections in patients receiving anti-TNF therapy reported to the 3-year prospective French RATIO registry. Ann. Rheum. Dis. 70, 616-623. doi: 10.1136/ard.2010. 137422

Scher, J. U., Ubeda, C., Equinda, M., Khanin, R., Buischi, Y., Viale, A., et al. (2012). Periodontal disease and the oral microbiota in new-onset rheumatoid arthritis. Arthritis Rheum. 64, 3083-3094. doi: 10.1002/art.34539

Schoels, M., Aletaha, D., Smolen, J. S., and Wong, J. B. (2012). Comparative effectiveness and safety of biological treatment options after tumour necrosis factor alpha inhibitor failure in rheumatoid arthritis: systematic review and indirect pairwise meta-analysis. Ann. Rheum. Dis. 71, 1303-1308. doi: 10.1136/annrheumdis-2011-200490

Schweinhardt, P., Kalk, N., Wartolowska, K., Chessell, I., Wordsworth, P., and Tracey, I. (2008). Investigation into the neural correlates of emotional augmentation of clinical pain. Neuroimage 40, 759-766. doi: 10.1016/j.neuroimage. 2007.12.016

Scott, D. L., Wolfe, F., and Huizinga, T. W. (2010). Rheumatoid arthritis. Lancet 376, 1094-1108. doi: 10.1016/S0140-6736(10)60826-4 
Senaldi, G., Yin, S., Shaklee, C. L., Piguet, P. F., Mak, T. W., and Ulich, T. R. (1996). Corynebacterium parvum- and Mycobacterium bovis bacillus Calmette-Guerin-induced granuloma formation is inhibited in TNF receptor I (TNF-RI) knockout mice and by treatment with soluble TNF-RI. J. Immunol. 157, 5022-5026.

Serrats, J., and Sawchenko, P. E. (2009). How T-cell-dependent and -independent challenges access the brain: vascular and neural responses to bacterial lipopolysaccharide and staphylococcal enterotoxin B. Brain Behav. Immun. 23, 1038-1052. doi: 10.1016/j.bbi.2009.06.004

Skelly, D. T., Hennessy, E., Dansereau, M. A., and Cunningham, C. (2013). A systematic analysis of the peripheral and CNS effects of systemic LPS, IL1beta, [corrected] TNF-alpha and IL-6 challenges in C57BL/6 mice. PLoS ONE 8:e69123. doi: 10.1371/journal.pone.0069123

Smolen, J. S., Landewe, R., Breedveld, F. C., Buch, M., Burmester, G., Dougados, M., et al. (2014). EULAR recommendations for the management of rheumatoid arthritis with synthetic and biological disease-modifying antirheumatic drugs: 2013 update. Ann. Rheum. Dis. 73, 492-509. doi: 10.1136/annrheumdis-2013204573

Sokka, T., Toloza, S., Cutolo, M., Kautiainen, H., Makinen, H., Gogus, F., et al. (2009). Women, men, and rheumatoid arthritis: analyses of disease activity, disease characteristics, and treatments in the QUEST-RA study. Arthritis Res. Ther. 11, R7. doi: 10.1186/ar2591

Steens, S. C., Steup-Beekman, G. M., Bosma, G. P., Admiraal-Behloul, F., Olofsen, H., Doornbos, J., et al. (2005). The effect of corticosteroid medication on quantitative MR parameters of the brain. AJNR Am. J. Neuroradiol. 26, 2475-2480.

Strangfeld, A., Listing, J., Herzer, P., Liebhaber, A., Rockwitz, K., Richter, C., et al. (2009). Risk of herpes zoster in patients with rheumatoid arthritis treated with anti-TNF-alpha agents. JAMA 301, 737-744. doi: 10.1001/jama.2009.146

Symmons, D. P., Bankhead, C. R., Harrison, B. J., Brennan, P., Barrett, E. M., Scott, D. G., et al. (1997). Blood transfusion, smoking, and obesity as risk factors for the development of rheumatoid arthritis: results from a primary care-based incident case-control study in Norfolk, England. Arthritis Rheum. 40, 1955-1961. doi: 10.1002/art.1780401106

Taylor, P. C. (2014). Aetiopathology of rheumatoid arthritis. Medicine 42, 227-230. doi: 10.1016/j.mpmed.2014.02.010

Teeling, J. L., Carare, R. O., Glennie, M. J., and Perry, V. H. (2012). Intracerebral immune complex formation induces inflammation in the brain that depends on Fc receptor interaction. Acta Neuropathol. 124, 479-490. doi: 10.1007/s00401012-0995-3

Thomson, C. A., McColl, A., Cavanagh, J., and Graham, G. J. (2014). Peripheral inflammation is associated with remote global gene expression changes in the brain. J. Neuroinflammation 11:73. doi: 10.1186/1742-2094-11-73

Tracey, D., Klareskog, L., Sasso, E. H., Salfeld, J. G., and Tak, P. P. (2008). Tumor necrosis factor antagonist mechanisms of action: a comprehensive review. Pharmacol. Ther. 117, 244-279. doi: 10.1016/j.pharmthera.2007.10.001

Tubach, F., Salmon, D., Ravaud, P., Allanore, Y., Goupille, P., Breban, M., et al. (2009). Risk of tuberculosis is higher with anti-tumor necrosis factor monoclonal antibody therapy than with soluble tumor necrosis factor receptor therapy: the three-year prospective French Research Axed on Tolerance of Biotherapies registry. Arthritis Rheum. 60, 1884-1894. doi: 10.1002/art.24632

Upadhyay, J., Baker, S. J., Rajagovindan, R., Hart, M., Chandran, P., Hooker, B. A., et al. (2013). Pharmacological modulation of brain activity in a preclinical model of osteoarthritis. Neuroimage 64, 341-355. doi: 10.1016/j.neuroimage.2012.08.084

van der Woude, D., Rantapaa-Dahlqvist, S., Ioan-Facsinay, A., Onnekink, C., Schwarte, C. M., Verpoort, K. N., et al. (2010). Epitope spreading of the anti-citrullinated protein antibody response occurs before disease onset and is associated with the disease course of early arthritis. Ann. Rheum. Dis. 69, 1554-1561. doi: 10.1136/ard.2009.124537 van Heemst, J., van der Woude, D., Huizinga, T. W., and Toes, R. E. (2014). HLA and rheumatoid arthritis: how do they connect? Ann. Med. 46, 304-310. doi: 10.3109/07853890.2014.907097

van Vollenhoven, R. F. (2010). New and future agents in the treatment of rheumatoid arthritis. Discov. Med. 9, 319-327.

Vincent, C., de Keyser, F., Masson-Bessiere, C., Sebbag, M., Veys, E. M., and Serre, G. (1999). Anti-perinuclear factor compared with the so called "antikeratin" antibodies and antibodies to human epidermis filaggrin, in the diagnosis of arthritides. Ann. Rheum. Dis. 58, 42-48. doi: 10.1136/ard.58.1.42

Wallis, R. S., Broder, M. S., Wong, J. Y., Hanson, M. E., and Beenhouwer, D. O. (2004). Granulomatous infectious diseases associated with tumor necrosis factor antagonists. Clin. Infect. Dis. 38, 1261-1265. doi: 10.1086/ 383317

Wartolowska, K., Hough, M. G., Jenkinson, M., Andersson, J., Wordsworth, B. P., and Tracey, I. (2012). Structural changes of the brain in rheumatoid arthritis. Arthritis Rheum. 64, 371-379. doi: 10.1002/art.33326

Wegner, N., Wait, R., Sroka, A., Eick, S., Nguyen, K. A., Lundberg, K., et al. (2010). Peptidylarginine deiminase from Porphyromonas gingivalis citrullinates human fibrinogen and alpha-enolase: implications for autoimmunity in rheumatoid arthritis. Arthritis Rheum. 62, 2662-2672. doi: 10.1002/art.27552

Wellcome Trust Case Control Consortium. (2007). Genome-wide association study of 14,000 cases of seven common diseases and 3,000 shared controls. Nature 447, 661-678. doi: 10.1038/nature05911

Wendler, J., Hummel, T., Reissinger, M., Manger, B., Pauli, E., Kalden, J. R., et al. (2001). Patients with rheumatoid arthritis adapt differently to repetitive painful stimuli compared to healthy controls. J. Clin. Neurosci. 8, 272-277. doi: 10.1054/jocn.1999.0775

Weyand, C. M., and Goronzy, J. J. (1990). Disease-associated human histocompatibility leukocyte antigen determinants in patients with seropositive rheumatoid arthritis. Functional role in antigen-specific and allogeneic T cell recognition. J. Clin. Invest. 85, 1051-1057. doi: 10.1172/JCI114535

Wolfe, F., Michaud, K., and Chakravarty, E. F. (2006). Rates and predictors of herpes zoster in patients with rheumatoid arthritis and non-inflammatory musculoskeletal disorders. Rheumatology 45, 1370-1375. doi: 10.1093/rheumatology/kel328

Woolf, C. J. (1983). Evidence for a central component of post-injury pain hypersensitivity. Nature 306, 686-688. doi: 10.1038/306686a0

Zivadinov, R., Shucard, J. L., Hussein, S., Durfee, J., Cox, J. L., Bergsland, N., et al. (2013). Multimodal imaging in systemic lupus erythematosus patients with diffuse neuropsychiatric involvement. Lupus 22, 675-683. doi: $10.1177 / 0961203313486193$

Conflict of Interest Statement: The authors declare that the research was conducted in the absence of any commercial or financial relationships that could be construed as a potential conflict of interest.

Received: 01 September 2014; accepted: 17 October 2014; published online: 06 November 2014.

Citation: Fuggle NR, Howe FA, Allen RL and Sofat N (2014) New insights into the impact of neuro-inflammation in rheumatoid arthritis. Front. Neurosci. 8:357. doi: 10.3389/fnins.2014.00357

This article was submitted to Neuroendocrine Science, a section of the journal Frontiers in Neuroscience.

Copyright () 2014 Fuggle, Howe, Allen and Sofat. This is an open-access article distributed under the terms of the Creative Commons Attribution License (CC BY). The use, distribution or reproduction in other forums is permitted, provided the original author(s) or licensor are credited and that the original publication in this journal is cited, in accordance with accepted academic practice. No use, distribution or reproduction is permitted which does not comply with these terms. 